Revue d'histoire de l'Amérique française

REVUE D.HISTOIRE DE L'AMÉRIQUE FRANÇAISE

\title{
Sur le chemin du paradis : les joies d'aimer, de croire et de s’accomplir de Marie-Louise Globensky (1849-1919)
}

\section{Sophie Doucet}

Volume 70, numéro 3, hiver 2017

URI : https://id.erudit.org/iderudit/1039516ar

DOI : https://doi.org/10.7202/1039516ar

Aller au sommaire du numéro

Éditeur(s)

Institut d'histoire de l’Amérique française

ISSN

0035-2357 (imprimé)

1492-1383 (numérique)

Découvrir la revue

Citer cet article

Doucet, S. (2017). Sur le chemin du paradis : les joies d'aimer, de croire et de s'accomplir de Marie-Louise Globensky (1849-1919). Revue d'histoire de l'Amérique française, 70(3), 5-29. https://doi.org/10.7202/1039516ar
Résumé de l'article

Le journal personnel de Marie-Louise Globensky (1849-1919), qui couvre trois décennies de sa vie adulte, reflète un paysage émotionnel contrasté, oscillant entre souffrances et joies. Cet article s'intéresse aux joies de la diariste, qui se manifestent dans des circonstances précises, en particulier lorsqu'elle accomplit la mission dont elle se sent investie comme femme, comme catholique et comme bourgeoise, et qu'elle se sent cheminer vers le paradis. En étudiant les joies d'une diariste, cet article veut jeter un éclairage nouveau sur sa vision du monde et ses motivations, mais aussi sur les rapports de force à l'oeuvre dans la société montréalaise dont elle fait partie. 


\section{Sur le chemin du paradis : les joies d'aimer, de croire et de s'accomplir de Marie-Louise Globensky $($ I849-г9I9)}

SOPHIE DouceT

Université du Québec à Montréal

RÉSUMÉ • Le journal personnel de Marie-Louise Globensky (1849-1919), qui counre trois décennies de sa vie adulte, reflète un paysage émotionnel contrasté, oscillant entre souffrances et joies. Cet article sintéresse aux joies de la diariste, qui se manifestent dans des circonstances précises, en particulier lorsqu'elle accomplit la mission dont elle se sent investie comme ferme, comme catholique et comme bourgeoise, et qu'elle se sent cheminer vers le paradis. En étudiant les joies d'une diariste, cet article veut jeter un éclairage nouveau sur sa vision du monde et ses motivations, mais aussi sur les rapports de force à l'œunre dans la société montréalaise dont elle fait partie.

ABStract • Marie-Louise Globenskys (1849-1919) diary, which covers three decades of her adult life, reflects a contrasted emotional landscape, fluctuating between suffering and joy.The present article addresses the diarist's joy, which occurred in specific circumstances, especially when she was fulfilling the duty she felt to be her own as a catholic, bourgeois woman and felt herself to be on a path towards paradise. By studying the joys of the diarist, this article intends to shed new light on Globenskys vision of the world and on her motivations, as well as on the power relationships in the Montréal society to which she belonged.

$\mathrm{A}_{\text {ans, prend le temps de consigner dans son journal personnel les }}$

1. J'aimerais remercier Magda Fahrni, Denyse Baillargeon, Valérie Poirier, Isabelle Bouchard, Laurie Laplanche, Jacinthe Archambault et François Riffaud pour nos discussions autour de la joie de Globensky, pour vos lectures attentives de versions anciennes et récentes de ce travail et pour vos conseils éclairés. Merci aussi aux évaluateurs anonymes de la RHAF et à l'équipe de rédaction. 
événements et émotions de sa journée, principalement la joie d'avoir retrouvé, à la gare, en matinée, sa fille Justine et son gendre Louis ${ }^{2}$, revenant d'un long voyage sur la côte ouest américaine:

$[\ldots]$ à $8 \mathrm{~h} 45$, je partis avec mon mari pour la gare Windsor. Il y avait là Marie, Jeanne, $[\ldots]$. Ce fut une véritable ovation. Quelle joie de revoir nos enfants partis depuis trois mois et demi. Justine et Louis ont vraiment l'air très bien. Après les souhaits de bienvenue [...] [je] suis montée dans l'auto de Justine $[\ldots]$ pour nous rendre à Outremont. [...] Nous avons beaucoup causé jusqu’au lunch ${ }^{3}$.

La bourgeoise Marie-Louise Globensky (1849-1919), épouse de sir Alexandre Lacoste, est connue de l'historiographie comme une mère de famille dévouée, et comme une catholique dévote très engagée dans des organisations de charitét. Elle est aussi décrite comme une diariste prolifique, qui a fait la part belle, dans ses écrits, à la douleur et au chagrin. «La conviction que la vie est une vallée de larmes, surtout pour les épouses et pour les mères, semble avoir été profondément intériorisée par cette femme de la bourgeoisie montréalaise», écrit l'historienne Christine Hudon'5.

Bien que la souffrance soit effectivement une part importante de son expérience émotionnelle telle qu'elle la représente dans son journal, cet article montrera que Marie-Louise Globensky a aussi vécu et exprimé au cours de sa vie beaucoup de joies, comme l'évoque l'entrée du journal citée plus haut. En effet, la joie, tout autant que la souffrance, sont valorisées dans sa vision catholique du monde, la première lui donnant un avant-goût du paradis, la seconde lui en ouvrant les portes. «[...] [t]oujours je sens mon âme se balancer entre les joies et les peines ${ }^{6}{ }^{6}$, confie-t-elle à son journal en méditant devant la mer agitée, à Saint-Irénée, le 20 juillet 1903.

Cet article défend trois idées. Premièrement, que les joies de Globensky s'expriment quand elle perçoit que ses actions ou les événements qui surviennent sont en phase avec ses valeurs et ses buts, et qu'elle a l'impression de marcher vers le paradis. En fait, Globensky ressent surtout de

2. Justine Lacoste-Beaubien et Louis de Gaspé-Beaubien.

3. Journal, 27 avril 1917.

4. Voir Christine Hudon, «Des dames chrétiennes. La spiritualité des catholiques québécoises au XIX ${ }^{\mathrm{e}}$ siècle", Revue d'histoire de l'Amérique française, 49, 2 (automne 1995): 169-194; Patricia Smart, De Marie de l'Incarnation à Nelly Arcan. Se dire, se faire par l'écriture intime (Montréal, Boréal, 2014), 255 ; Yolande Pinard, «Les débuts du mouvement des femmes à Montréal, 1893-1902 », dans Marie Lavigne et Yolande Pinard, dir., Travailleuses et féministes (Montréal, Boréal, 1983), 184.

5. C. Hudon, loc. cit., 187.

6. Journal, 20 juillet 1903. 
la joie en se conformant au rôle qui est attendu d'elle, comme femme et mère, comme catholique et comme bourgeoise. Elle semble avoir parfaitement intériorisé les normes émotionnelles en cours dans sa "communauté émotionnelle ${ }^{7}$ ", la bourgeoisie franco-catholique montréalaise. Deuxièmement, ce n'est pas tout à fait candidement que Globensky exprime ses joies dans son journal. À travers cette représentation, elle a en effet une intention cachée: se poser en modèle pour ses enfants, à qui est destiné le journal ${ }^{8}$. Troisièmement, cet article propose qu'en valorisant certaines formes de joies, les normes émotionnelles en cours dans la bourgeoisie franco-catholique montréalaise sont au service du statu quo dans les rapports de pouvoir en place.

Notre étude s'inscrit dans la jeune historiographie des émotions, qui cherche à donner une place aux affects dans la compréhension que l'on se fait des sociétés du passé. Longtemps vue comme insaisissable, la dimension émotionnelle et affective du passé est en effet désormais considérée - jusqu'à un certain point - comme préhensible par les historiens des émotions ${ }^{9}$. Cet article s'alimente par ailleurs largement de la littéra-

7. Concept créé par Barbara Rosenwein, les «communautés émotionnelles» sont un peu les mêmes que les communautés familiales ou sociales, c'est-à-dire la famille, le voisinage, le parlement, le monastère, etc. À la différence que le chercheur qui s'y intéresse: «[...] seeks above all to uncover systems of feeling: what these communities (and the individuals within them) define and assess as valuable or harmful to them; the evaluations that they make about others' emotions; the nature of the affective bonds between people that they recognize; and the modes of emotional expression that they expect, encourage, tolerate, and deplore». B. H. Rosenwein, "Worrying about Emotions in History », American Historical Review, 107, 3 (juin 2002): 842.

8. Les cahiers manuscrits des années 1915, 1917 et 1919 portent, en page de garde, la mention explicite: «dédié à mes enfants".

9. Les travaux pionniers de Barbara Rosenwein et de William Reddy, qui se sont abreuvés aux découvertes des psychologues cognitivistes et des anthropologues, ont posé les bases théoriques rendant possible cette entreprise. L'histoire des émotions postule que les émotions ne sont pas un fait universel, commun à toutes les cultures, mais qu'elles ont une dimension sociale et culturelle forte. Elle s'oppose par ailleurs à une vision linéaire de l'évolution de l'émotivité en Occident, proposant une lecture plus morcelée. Pour elle, le rôle des émotions dans les processus de prises de décision des individus et des sociétés ne peut plus être inféodé à celui qui est joué par les idées ou la raison. Il participe d'une même entreprise et est tout aussi influent. Ne pas se pencher sur les émotions pour tenter de comprendre le passé, selon les historiens des émotions, serait donc s'empêcher de saisir des pans cruciaux de la réalité. Depuis 15 ans, l'histoire des émotions est florissante dans le monde anglo-américain et en France. Voir notamment: B. H. Rosenwein, loc. cit., 821-845 ; W. M. Reddy, The Navigation of Feeeling. A Framework for the History of Emotions (Cambridge, Cambridge University Press, 2001); Damien Boquet, «Faire l'histoire des émotions à l'âge des passions" (exposé présenté lors du Séminaire de recherche sur les îles britanniques: "Les émotions: performativité, pratiques, mises en scène»), EMMA. Les émotions au Moyen Âge, 15 novembre 2010, http://emma.hypotheses.org/1106 (consulté le 14 mai 2013); Piroska Nagy et Damien Boquet, "Historical Emotions, Historian's Emotions», Les émotions au Moyen Âge, 5 mars 2011, http://emma. hypotheses.org/ 1213 (consulté le 11 juillet 2013); Susan J. Matt, "Current Emotion Research in History: Or, Doing History from the Inside Out ", Emotion Review, 3,1 (janvier 2011): 117-12; Quentin Deluermoz, Emmanuel Fureix, Hervé Mazurel et M’hamed Oualdi, «Écrire l’histoire des émotions : de l’objet à la catégorie d'analyse ", Revue d'histoire du XIX' siècle, 47 (2013) : 155-189; Alain Corbin, Jean-Jacques Courtine 
ture autour des écrits de soi, qui montre que ces derniers ne sont jamais des témoignages innocents relayant la vérité nue, mais qu'ils sont écrits pour "agir sur le monde ${ }^{10}$ ». En analysant l'émotion qu'est la joie, nous souhaitons jeter un nouvel éclairage sur un individu - ses valeurs, sa vision du monde - et sur une «communauté émotionnelle», et ainsi contribuer à l'histoire sociale et culturelle du Québec ${ }^{11}$.

Avant de plonger dans le vif de l'analyse, nous présenterons brièvement Marie-Louise Globensky et la source qu'est son journal intime et nous proposerons une brève description historique de la joie. Puis, afin de défendre les trois idées énoncées précédemment, cette étude sera développée suivant trois parties principales, selon les types de joies exprimées par Globensky: les joies d'amour et de liens, les joies spirituelles et les joies de s'accomplir et de s'engager dans la société.

\section{MARIE-LOUISE GLOBENSKY ET SON JOURNAL}

Marie-Louise Globensky est la fille du percepteur des douanes Léon Globensky (1807-1879) et de Marguerite-Angélique Limoges (1814-1883) ${ }^{12}$; elle épouse en 1866 Alexandre Lacoste (1842-1923), qui sera avocat, professeur de droit, sénateur et juge en chef de la Cour du banc de la reine ${ }^{13}$.

et Georges Vigarello, Histoire des émotions. Tome 1. De l'Antiquité aux Lumières (Paris, Seuil, 2016). Alain Corbin, Jean-Jacques Courtine et Georges Vigarello, Histoire des émotions. Tome 2. Des Lumières à la fin du XIXe siècle (Paris, Seuil, 2016); Jan Plamper, The History of Emotions. An Introduction (Oxford, Oxford University Press, 2015).

10. Christian Jouhaud, Dinah Ribard et Nicolas Schapira. Histoire, littérature, témoignage. Écrire les malheurs du temps (Paris, Gallimard, 2009), 134. Le journal intime, reconnait par ailleurs cette historiographie, sert non seulement à cerner et à transformer le «soi» de la personne qui écrit, mais il contribue également à modifier la culture dans laquelle cette personne évolue. Voir Suzanne L. Bunkers et Cynthia A. Huff, dir., Inscribing the Daily. Critical Essays on Women's Diaries (Amherst, University of Massachusetts Press, 1996); Françoise Simonet-Tenant, Le journal intime. Genre littéraire et écriture ordinaire (Paris, Nathan, 2001); Jean-Pierre Bardet et François-Joseph Ruggiu, dir., Au plus près du secret des cours? Nouvelles lectures historiques des écrits du for privé en Europe du XVI a ${ }^{e}$ XVIII siècle (Paris, Presses de l'Université ParisSorbonne, 2005); Françoise Simonet-Tenant, Journal personnel et correspondance (1785-1939) ou les affinités électives (Louvain-La-Neuve, Bruylant-Academia, 2009).

11. Si l'histoire des émotions n'a pas encore fait de nombreux émules chez les historiens du Québec, les émotions comme l'angoisse, la tristesse, la culpabilité, la honte ont néanmoins été présentes, à divers degrés, dans plusieurs travaux en histoire sociale. Voir par exemple: Serge Gagnon, Plaisir d'amour et crainte de Dieu. Sexualité et confession au Bas-Canada (Sainte-Foy, Les Presses de l’Université Laval, 1990); Denyse Baillargeon, Ménagères au temps de la Crise (Montréal, Éditions du remue-ménage, 1991); Gérard Bouchard, «La sexualité comme pratique et rapport social chez les couples paysans du Saguenay (18601930)", Revue d'histoire de l'Amérique française, 54, 2 (automne 2000): 183-217; Magda Fahrni, "Glimpsing Working-Class Childhood through the Laurier Palace Fire of 1927: The Ordinary, the Tragic, and the Historian's Gaze», The Journal of the History of Childhood and Youth 8, 3 (automne 2015): 426-450.

12. Voir Yvon Globensky, Histoire de la famille Globensky (Montréal, Éditions du Fleuve et Yvon Globensky, 1991), 96-101.

13. Sur le plan idéologique, Alexandre Lacoste est considéré comme un conservateur modéré. Il joue un rôle de conseiller «influent, mais discret », auprès du Parti conservateur. Il est fait chevalier comman- 
Le couple aura treize enfants ${ }^{14}$, dont la féministe maternaliste Marie Gérin-Lajoie ${ }^{15}$ et la cofondatrice de l'hôpital pour enfants Sainte-Justine, Justine Lacoste-Beaubien ${ }^{16}$. Globensky tient un journal personnel extrêmement riche, commencé à 15 ans, délaissé peu avant son mariage à l'âge de 17 ans et repris à l'âge de 39 ans, jusqu'à la veille de sa mort, à 70 ans ${ }^{17}$. Il comprend vingt-cinq cahiers manuscrits ${ }^{18}$.

Ce journal - quotidien pendant de longues périodes - est d'abord une sorte de carnet de bord comprenant des informations sur les événements de sa journée, comme la messe, le marché et les rencontres sociales ou familiales. Mais à mesure qu'elle vieillit, et en fonction de la valeur émotive des événements, le journal devient aussi le confident de ses états d'âme ${ }^{19}$. On y retrouve de la tristesse face aux séparations, aux deuils, à la guerre et à la vieillesse; de l'angoisse face à la maladie de personnes chères et face aux changements sociaux, beaucoup d'amour pour ses proches et bien sûr de la joie, des émotions et des sentiments qui sont bien vus par

deur de l'ordre de Saint-Michel et Saint-Georges en 1892, ce qui lui vaut le droit de placer le prédicat honorifique sir devant son prénom. Voir: Sylvio Normand, "LACOSTE, sir ALEXANDRE», dans Dictionnaire biographique du Canada, vol. 15 (Université Laval/University of Toronto, 2003), www.biographi.ca/fr/bio/lacoste_alexandre_15F.html (consulté le 29 janv. 2017).

14. Marie (1867-1945), Louis (1869-1909), Henriette (1870-1871), Blanche (1872-1957), Paul (1874-1945), Justine (1877-1967), Jeanne (1879-1962), Yvonne (1881-1947), Alexandre (1883-1940), Arthur (1885-1888), Thaïs (1886-1962), Berthe (1889-1966), René (1891-1892). Dix de ces treize enfants ont survécu à la petite enfance.

15. Voir notamment: Anne-Marie Sicotte, Marie Gérin-Lajoie. Conquérante de la liberté (Montréal, remueménage, 2014). Hélène Pelletier-Baillargeon, Marie Gérin-Lajoie. De mère en fille, la cause des femmes (Montréal, Boréal Express, 1985); Karine Hébert, «Une organisation maternaliste au Québec: la Fédération nationale Saint-Jean-Baptiste et la bataille pour le vote des femmes ", Revue d'histoire de l'Amérique française, 52, 3 (hiver 1999): 315-344.

16. Voir notamment: Denyse Baillargeon, Naître, vivre, grandir: Sainte-Justine, 1907-2007 (Montréal, Boréal, 2007). Madeleine Des Rivières, Une femme, mille enfants: Justine Lacoste Beaubien, 1877-1967 (Montréal, Bellarmin, 1987); Nicolle Forget, Francine Harel Giasson et Francine Séguin, Justine LacosteBeaubien et l'hôpital Sainte-Justine (Sainte-Foy, Presses de l’Université du Québec, 1995).

17. Son journal est représentatif des journaux féminins de l'époque, qui suivent les cycles de la vie des femmes, laissant de grands blancs pour les périodes où la maternité occupe tout l'espace et le temps. Voir: Amanda Vickery, “"S'il vous plaît, brûlez cela afin qu'aucun œil mortel ne puisse le voir” : Les secrets des sources féminines », dans J.-P. Bardet et F.-J. Ruggiu, dir. Au plus près du secret des cours ?, op. cit, 57.

18. Il est disponible à BAnQ Vieux-Montréal, Fonds Famille Lacoste (P76). Le journal est aussi disponible sous la forme d'un tapuscrit, dans le même Fonds d'archives. Il a en effet été dactylographié par la secrétaire de Paul Lacoste, un des fils de Marie-Louise Globensky, peu après le décès de la diariste, dans les années 1920. Il se présente en six volumes totalisant environ 2500 pages, format légal ( 8 1 $1 / 2 \mathrm{x} 14)$, sur du papier pelure (très mince). Il existe en quelques copies qui appartiennent à des descendants de la famille.

19. Son journal suit en cela l'évolution de l'histoire de la pratique du journal intime. En effet, apparue à mesure que s'imposait le concept d'intimité au XVIII ${ }^{\mathrm{e}}$ siècle, l'habitude de consigner pour soi son quotidien se développe pour devenir assez commune au sein des classes aisées dans le XIX $\mathrm{X}^{\mathrm{e}}$ siècle et, de plus en plus, s'intéressera à décrire les émotions et l'intériorité, plutôt que les événements du quotidien vécus par celui ou celle qui tient la plume. Voir: Françoise Simonet-Tenant, Journal personnel et correspondance (1785-1939) ou les affinités électives (Louvain-La-Neuve, Academia Bruylant, 2009), 28-35. Notre analyse se concentrera sur les années 1897 à 1919 du journal, qui sont plus riches en représentations émotionnelles. 
la communauté émotionnelle ${ }^{20}$. Les émotions représentées dans le journal de Globensky reflètent sans aucun doute l'adhésion très forte de cette femme aux enseignements de l'Église catholique, qui ont su rendre son existence - malgré les épreuves et la souffrance - pleine de sens et parsemée de moments heureux.

\section{LA JOIE}

Les historiens se sont davantage intéressés aux émotions que sont la colère, la peur et le chagrin qu'à la joie, perçue dans les sciences sociales comme une émotion puérile, candide, qui n'a pas la noblesse de la souffrance ${ }^{21}$. Pour l'historien américain Darrin McMahon, cela s'explique peut-être par l'enracinement de l'histoire des émotions dans l'histoire sociale, qui s'intéresse surtout aux exclus, aux opprimés et aux rapports de pouvoir, des questions qui génèrent davantage d'émotions "négatives» que "positives $»^{22}$.

Comme les autres émotions, la joie apparaît en fonction d'un processus de perception et d'évaluation de la réalité, guidée par une vision du monde personnelle et culturelle, considèrent aujourd'hui les psychologues et neuroscientifiques ${ }^{23}$. Davantage qu'une absence d'émotion douloureuse, la joie recouvre toute une palette d'expériences agréables: la gratitude, l'émerveillement, l'enthousiasme, l'inspiration, la curiosité, le plaisir, la fierté, la satisfaction, etc. Elle joue un rôle dans l'adaptation des personnes aux circonstances de leur vie; elle améliore la résilience et la créativité et aide à gérer l'adversité, considère-t-on ${ }^{24}$. Pour le psychologue Bernard Rimé, la

20. Cet article, qui se concentre sur la joie, est issu d'un projet plus vaste, notre thèse de doctorat en cours, qui embrasse l'ensemble du paysage émotionnel de Globensky.

21. Les émotions positives sont associées avec la complaisante et l’hédonisme de la société de consommation, écrit l'historien Darrin McMahon. «Happy are those who hava a coke and a smile, not who read Kafka or Sartre or contemplate the Shoah or the depths of the poetry of Sylvia Plath ", D. McMahon, "Finding Joy in the History of Emotions», dans Susan J. Matt and Peter N. Stearns, dir., Doing Emotions History (Chicago, University of Illinois Press, 2014), 107.

22. D. McMahon, op.. cit., 105.

23. Selon les psychologues cognitivistes, les émotions sont des processus de perception et d'évaluation de la réalité entraînant des manifestations psychiques et physiques. Voir notamment: Joseph Ledoux, Le cerveau des émotions (Paris, Odile Jacob, 2005); Silvie Berthoz et Silvia Krauth-Gruber, La face cachée des émotions (Paris, Éditions Le pommier, 2011). Gardons toutefois en tête que ce savoir «scientifique» doit être considéré comme en constante évolution et appartenant à une époque, non comme «la» vérité. Voir sur ce sujet la réflexion stimulante de Damien Boquet et Piroska Nagy, «Pour une histoire intellectuelle des émotions. Introduction ", L'atelier du Centre de recherches historiques [en ligne], 16, 2016, mis en ligne le 23 mai 2016 (consulté le 7 juin 2016).

24. Voir notamment, Barbara L. Fredrickson, "The Role of Positive Emotions in Positive Psychology : The Broaden-and-Build Theory of Positive Emotions », American Psychologist, 56, 3 (mars 2001): 218-226; Alice Isen, "Positive Affect and Decision Making», dans M.Lewis and J. Haviland, dir., Handbook of Emotions (New York, Guilford Press, 2000), 261-277. 
joie est l'émotion qui marque l'atteinte du but ou les phases d'atteinte du but ${ }^{25}$.

Les neuroscientifiques ne sont pas les premiers à avoir compris l'importance de cette émotion. La joie était déjà valorisée dans l'Antiquité préchrétienne et chrétienne ${ }^{26}$, chez les penseurs chrétiens du Moyen Âge ${ }^{27}$ et son importance aurait été renforcée lors de la réforme protestante, alors qu'elle est devenue une sorte de devoir religieux, une preuve de foi, quand la tristesse devenait pour les protestants une émotion honteuse ${ }^{28}$. Depuis le $\mathrm{XVI}^{\mathrm{e}}$ siècle, la conception de la joie des protestants aurait évolué dans des directions différentes selon les confessions, mais la jovialité (ou joie affichée) serait demeurée une caractéristique des protestants états-uniens au cours du $\mathrm{XX}^{\mathrm{e}}$ siècle $^{29}$.

Chez les catholiques, tout au long de l'histoire, la joie est valorisée, comme la souffrance ( Jesus, the man of sorrows, is at the same time a fountain of joy $\left.^{30} \ldots »\right)$; toutes ces émotions participant à ouvrir les portes du paradis. En effet, les émotions des catholiques sont évaluées en fonction du but suprême: atteindre le bonheur, non sur cette terre, mais au ciel. Par ailleurs, dans l'histoire chrétienne, la joie est une perfection, elle est donc de nature divine, au contraire des émotions de souffrance qui sont imperfections, donc de nature humaine ${ }^{31}$.

\section{LES JOIES DE MARIE-LOUISE GLOBENSKY}

\section{Les joies d'amour et de liens}

La joie qui apparaît le plus fréquemment dans le journal intime de Globensky est sûrement la joie d'aimer et d'être aimée. En effet, en avançant dans la vie entourée d'affection, maillée aux autres, principalement

25. Bernard Rimé, Le partage social des émotions (Paris, Presses universitaires de France, 2005), 32-34.

26. Adam Potkay, The Story of Joy. From the Bible to the late Romantism (Cambridge, Cambridge University Press), 39-49. Voir aussi pour la présence de la joie dans l'Ancien et le Nouveau Testament: Robert Larger, La joie dans sa plénitude. Essai sur la joie chrétienne (Paris, Téqui, 1988), 247-269. La notion de joie et de bonheur apparaît 230 fois dans le Nouveau Testament et plus de 800 fois dans l'ensemble de la Bible, montre Larger, ibid., 92-94.

27. A. Potkay, op. cit., 50-72.

28. " $[\ldots]$ this Protestant moralisation of mood had the curious consequence of both sanctioning joy and happiness as presumptive signs of God's grace and of increasing anxiety about their attainment. If good Christians should feel joy and ought to experience happiness in the daily life, it followed ineluctably that failure to do so was to be doubtly damned», D. McMahon, op. cit, 113.

29. Voir Christina Kotchemidova, "From Good Cheer to "Drive-By Smiling" : A Social History of Cheerfulness", Journal of Social History, 39 (2005): 5-37 et Peter N. Stearns American Cool: Constructing a Twentieth-Century Emotional Style (New York, New York University Press, 1994).

30. D. McMahon, op. cit, 112.

31. R. Larger, op. cit. 120-121. 
à son mari, ses enfants, ses frères et sœurs et ses amies, elle se sent accomplir sa principale raison d'être sur terre et marcher sur le chemin du bonheur céleste. Car en aimant, elle respecte le premier commandement des Évangiles: «Voici quel est mon commandement: vous aimer les uns les autres comme je vous ai aimés», a dit Jésus ${ }^{32}$.

\section{Joies douces du quotidien}

Le quotidien de Marie-Louise Globensky est rempli de rencontres avec des membres de sa famille ou des amies, qui souvent lui font ressentir de la joie.

Par exemple, le 3 décembre 1898, à 49 ans, Globensky passe une journée "ordinaire» avec plusieurs de ses proches: ses enfants, petits-enfants et son mari. L’entrée de son journal laisse voir les émotions ressenties:

Ce matin je suis allée voir mes enfants et petits-enfants. D’abord chez Louis. Le petit couple est à peindre tant ils sont jolis à voir. De là chez Blanche ${ }^{33}$, second tableau également cher à mon cœur, Louise et René superbes et à croquer. Puis ensuite je me rendis chez Marie où les quatre chérubins m'attendaient encore en me tendant les bras $^{34}$. Quel bonheur je ressens en les voyant tous si bien portants et si charmants, je ne saurai jamais à qui donner la palme mais tout ce que je sais c'est qu'ils occupent une large place dans mon cœur. J’ai pris le lunch à cette troisième visite, ce qui parut enchanter mon petit monde puis je revins ensuite à la maison. À $3 \mathrm{~h} 30$, mon mari arriva de Québec et nous eûmes le plaisir d'une soirée de causerie très agréable ${ }^{35}$.

Le 31 janvier 1918, elle passe un après-midi tranquille et heureux avec sa sœur Élodie et sa nièce Minette. «Ma sœur Élodie et Minette Archer sont venue $[$ sic $]$ prendre le dîner avec nous. Ce fut bien agréable de causer tout l'après-midi avec notre petit ouvrage, dans une si parfaite intimité ${ }^{36}$." Globensky, on le voit, valorise la qualité des liens, chérit l’intimité et la complicité avec ces personnes qui lui sont chères, dont aussi ses amies proches. Le 17 mai 1916 son amie d'enfance Blanche Wotherspoon ${ }^{37}$ vient

32. "Aimer Dieu, s'aimer soi-même, aimer son prochain, aimer ses ennemis : tel est l'ordre - à la fois l'injonction et la hiérarchie - de l'amour qui doit régir la vie sociale et le lien à Dieu ", Damien Boquet et Piroska Nagy, Sensible Moyen Âge. Une histoire des émotions dans l'Occident médiéval (Paris, Seuil, 2015), 23.

33. Louis et Blanche Lacoste, fils et fille de Globensky, sont voisins. En effet, selon l'annuaire Lovell, Louis Lacoste habite au 407 de la rue Saint-Hubert, entre Sherbrooke et Cherrier et Blanche LacosteLandry habite au 409 de la rue Saint-Hubert. Globensky, elle, est au 71 de la rue Saint-Hubert, près de Dorchester.

34. Marie Gérin-Lajoie habite au 577 Sherbrooke, près de Saint-Urbain.

35. Journal, 3 décembre 1898.

36. Journal, 31 janvier 1918.

37. Blanche Juchereau Wotherspoon est née à Saint-Ours en 1848 et morte à Londres en 1924. Elle est la fille de Louis-Charles Juchereau et d'Harriette Maria Duchesnay et l'épouse de Ivan Tolkien Wotherspoon. 
luncher avec elle. Dans son journal, Globensky, 67 ans, écrit: «J’ai eu aujourd'hui à luncher mon amie madame Wotherspoon. Nous avons eu une conversation bien intime que de souvenirs nous avons évoqués ${ }^{38}$.»

Ce bonheur de passer du temps avec les êtres aimés, Globensky le vit aussi en vacances, dans le calme des grands espaces, comme le 25 août 1905, à Saint-Irénée, dans Charlevoix :

Une journée idéale. Je passe la matinée sur la grève avec mon mari, nous laissant caresser par la brise et ne nous lassant pas d'admirer cette nature si grandiose qui parle tant à l'âme. Dans l'après-midi, je retourne encore, et cette fois c'est avec ma bonne amie Mme Frémont. Nous apportons notre ouvrage avec un livre bien intéressant et à tour de rôle nous lisons pendant que l'autre agite ses doigts sur l'aiguille qu'elle dirige. Qu'il est bon de se retrouver ainsi dans un profond silence loin de toute agitation mondaine. Nous voyons en face de nous notre fraîche jeunesse sur la verte pelouse, jetant et recevant des balles de tennis. Un autre groupe à robes blanches et roses assises au pied des arbres, les regardent, nous paraissant être une corbeille de fleurs. Que je suis reconnaissante à Notre Seigneur de nous réserver des heures si délicieuses ${ }^{39}$.

Sa joie de passer du temps avec les personnes qu'elle aime au quotidien est souvent accompagnée de gratitude envers Dieu qui rend possible ces moments. Elle ne se lasse pas de la décrire, parce qu'elle est bien vue, et lui permet de passer un message aux destinataires du journal sur l'importance de la qualité des liens affectifs.

\section{Joies fortes des jours de fêtes et d'anniversaires}

La joie d'être ensemble s'exprime avec plus d'emphase dans le journal lors de fêtes et d'anniversaires. Au point où elle apparait parfois fabriquée et au service des intentions de l'écriture (montrer la voie à ses enfants) plus que collée sur le ressenti. Par exemple, d'une année à l'autre, les descriptions des fêtes du Nouvel An se ressemblent de façon étonnante. Le 31 décembre 1898, âgée de 49 ans, elle écrit:

[...] Ce soir, nous avons notre ravissante fête de famille. Dès sept heures du soir, tous mes enfants et petits-enfants arrivent gaiement et se placent autour de la grande table pour attendre les étrennes. [...] Rien de plus charmant que cette heure où tous ne semblent avoir qu'un cour c'est l'expression d'un bonheur qui ne peut se peindre pour ceux qui reçoivent comme pour ceux qui donnent. Quel touchant spectacle, qu'elles sont douces ces joies pures 
que l'on goûte au foyer, si plein d'affections tendres, puissions-nous y revenir pendant bien des années encore et n'y voir que des sourires et des joies ${ }^{40}$.

Vingt ans plus tard, l'entrée, semblable, est teintée de plus de gratitude:

Le dîner fut charmant, d'une grande gaité. [...] Nous avons ensuite fait la distribution générale des étrennes, ce qui fait toujours le bonheur de chacun. La soirée fut donc charmante et nous en conserverons un bien doux souvenir. Quel suave bonheur de se voir entourés ainsi de nos chers enfants rien n'est comparable à ces fêtes familiales où le cour déborde d'une joie si pure et si vraie, merci mon Dieu de ces heures précieuses ${ }^{41}$.

En 1898 comme en 1919, donc, la fête familiale du Nouvel An s'est caractérisée, selon la représentation qu'elle en fait, par la gaité, le bonheur, l'affection, les «joies pures» d'être en famille. Dans l'analyse d'écrits de soi, la répétition doit toujours attirer l'attention ${ }^{42}$. Ici, elle est probablement révélatrice de ce que Globensky croit que doit être une soirée du Nouvel An réussie, c'est-à-dire une soirée pleine d'harmonie et de bonheur, plus qu'elle ne décrit la soirée telle qu'elle s'est exactement déroulée. Cela ne veut pas dire que l'émotion décrite est fausse, mais il est envisageable que la diariste puisse omettre des informations allant à l'encontre de ses valeurs personnelles et de la norme.

Par ailleurs, les silences sont parlants. Si, dans les descriptions de fêtes que fait Globensky, l'on retrouve parfois de la tristesse ou de l'angoisse à cause d'un absent ou d'un souci dans la famille, jamais il n'est question de tension, de conflit, de jalousie, de colère, de désamour. Le silence complet sur ces émotions nous renseigne sur le fait que Globensky les considère honteuses, peu acceptables ${ }^{43}$. En effet, elles font pour elle obstacle à l'harmonie familiale. Alors que la joie dans une fête de famille est, elle, une fierté, une preuve de l'harmonie (par elle et par Dieu?) réalisée.

Au cours des fêtes, l'absence d'une seule personne ternira immanquablement la joie de Globensky, comme si la présence de tous était, plus que toute autre, une preuve de son devoir de mère accompli. Ainsi, la joie de

40. Journal, 31 décembre 1898.

41. Journal, $1^{\mathrm{er}}$ janvier 1919.

42. «Private writings (...) ask of us, if we wish to read them knowingly, a special inventive patience. We must interpret what is not written as well as what is, and, rather than dismiss repetition, value them especially », Elizabeth Hampsten, citée dans S. Bunkers et C. Huff, op. cit., 11.

43. D’ailleurs, si Jésus est «[...] parcouru d'émotions qu'il ne cherche ni à cacher ni à neutraliser car elles sont les signes de son humanité", il n'éprouve "point de jalousie, d'envie ou de haine, mais des émotions vertueuses qui peuvent contribuer au salut et à la juste colère», D. Boquet et P. Nagy, Sensible Moyen Âge..., op. cit., 22. 
Globensky est "complète» ou "parfaite» lorsque tous les siens sont là, comme le lundi $1^{\text {er }}$ janvier 1900 :

Nous avons reçu un bon nombre de visiteurs cet après-midi, puis nous avons eu notre grand dîner de famille ce soir, il ne manquait personne, aussi ai-je joui d'un bonheur parfait. Entourée de tous mes enfants, rien de meilleur sur la terre, d'aussi suave que ces réunions intimes où tous les cœurs battent à l'unisson et sont heureux de se rencontrer ${ }^{44}$.

Ce sentiment de joie face au devoir familial accompli est particulièrement palpable dans l'entrée du 8 mai 1916, jour du 50 anniversaire de mariage de Marie-Louise Globensky et d'Alexandre Lacoste. L'entrée mérite d'être citée longuement:

Le voici donc ce grand jour auquel nous nous préparons depuis si longtemps. Le ciel est pur et d'un bleu d'azur, la température est bonne, tout présage un jour de fête. Aussi fut-il délicieux au-delà de mes espérances, j'en fus saisie de poignantes émotions. À 9 heures nous étions tous rendus à la chapelle de N. D. de Lourdes parée de ses plus beaux atours, resplendissante de lumières et de fleurs. La musique nous réjouit de ses plus mélodieux accords. L’allocution de Mr le curé Gauthier, des plus touchantes. Lorsqu'à la communion nous nous approchions de la table sainte, mon mari et moi suivis de nos enfants et petitsenfants, mon cœur déborda de reconnaissance pour N.S. [Notre seigneur] [...]. Quel suave souvenir nous garderons de cette si belle cérémonie. Au retour à la maison où nous étions à peu près 80 , la gaité régna, tout le monde semblait être content. Nous prîmes un copieux déjeuner, tous sur des tables de quatre personnes. Nous avions l'honneur d'avoir avec nous l'archevêque Mgr Bruchési, Mgr Gauthier, son coadjuteur le curé de Saint-Jacques, puis l'abbé Archambault. Mgr Bruchési nous avait fait venir la bénédiction du pape. J'espère qu'elle nous portera bonheur ainsi qu'à notre grande famille. Dans l'après-midi nous avons fait photographier le groupe familial dans le jardin, 44, c'est assez exceptionnel. Le soir, grand dîner plus intime, puis très intéressante soirée où nos chers enfants nous avaient réservé bien des surprises. [...] Enfin, jamais nous pourrons oublier la journée du 8 mai 1916. Jour de reconnaissance et action de grâce ${ }^{45}$.

Validée par la religion (une messe, une bénédiction papale, un archevêque, un évêque et deux prêtres présents à la réception), la joie de cette journée est bel et bien celle du devoir accompli. Elle souligne l'union réussie et féconde d'un homme et d'une femme sous le regard de Dieu. Elle est colorée par la gratitude que dit ressentir Globensky envers Dieu, 


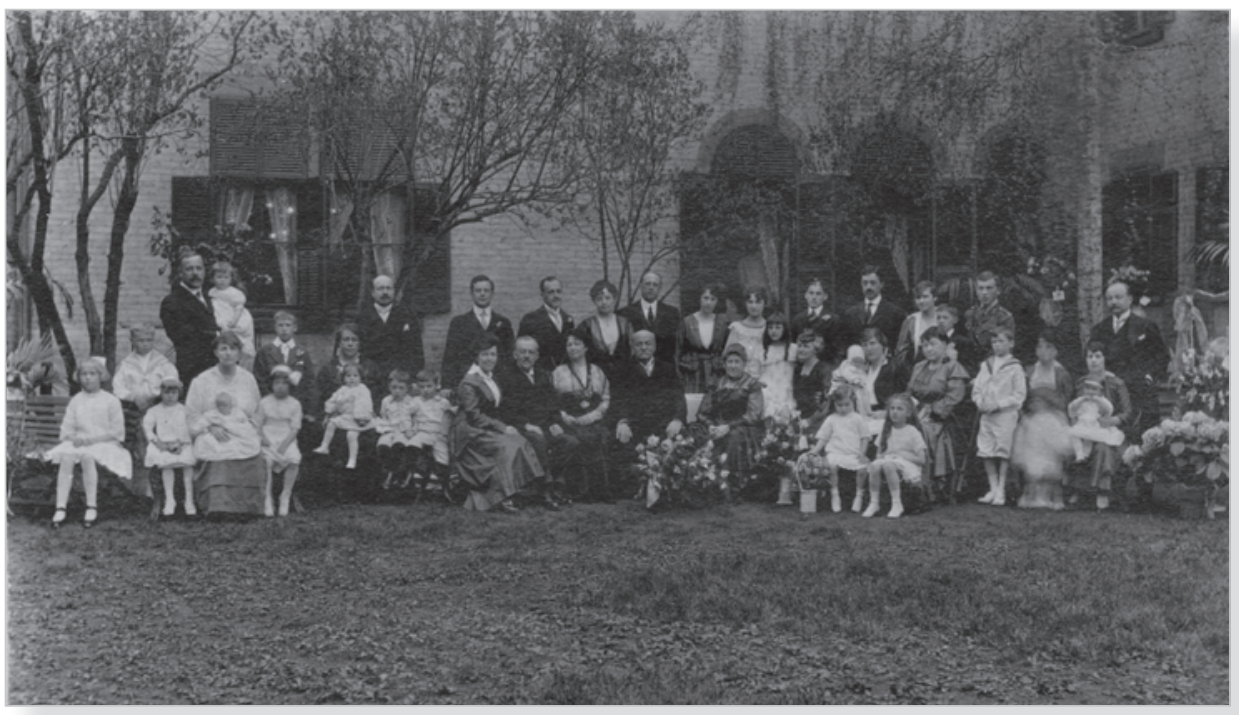

Photo de famille prise le 8 mai 1916, lors des noces d'or de Marie-Louise Globensky et Alexandre Lacoste. À son propos, on peut lire dans le journal Globensky: «Dans l'aprèsmidi, nous avons fait photographier le groupe familial dans le jardin, 44, c'est assez exceptionnel », Journal, 8 mai 1916.

Source: BAnQ Vieux-Montréal, Fonds famille Landry, PI55, SI, SS2, D33, P3.

d'avoir pu conduire jusque-là le bateau de cette grande famille unie et harmonieuse (du moins en apparence). Par ailleurs, elle montre l'exemple à ceux qui liront le journal.

\section{Joie intense des retrouvailles}

Là où la joie d'être ensemble se fait la plus intense et s'exprime avec le plus d'effusion, notamment par la description de gestes de joie, c'est lors de retrouvailles avec les personnes aimées. Par exemple, le 28 août 1904, de retour de vacances à Saint-Irénée, Globensky écrit: «[...] nous trouvons à la gare Marie et tout son monde pour nous sauter au $\operatorname{cou}^{46}$ ».

Les retrouvailles avec ses filles adultes sont des événements assez fréquents, parce que quelques-unes d'entre elles habitent dans des villes éloignées: Thaïs et Blanche à Québec, Yvonne à Rimouski. En avril 1908, un an après son mariage avec Auguste-Maurice Tessier, Yvonne (la $8^{\mathrm{e}}$ enfant de la famille) revient passer quinze jours à la maison familiale avec son nouveau-né. «Ce soir c’est l’arrivée de ma chère Yvonne avec son bébé et sa

46. Journal, 28 août 1904. 
bonne, quelle joie de l'avoir pour quelque temps avec nous ${ }^{47}$ ", écrit Globensky. Les séjours de ses filles à la maison familiale seront souvent vécus dans un mélange de joie et de nostalgie de l'époque où elles vivaient encore à la maison. Globensky se donne alors l’illusion de les «posséder encore ».

Lorsque les retrouvailles sont une surprise ou que la personne retrouvée est quelqu'un qu'elle n'a pas vu depuis très longtemps, les émotions sont encore plus vives et leurs manifestations physiques plus souvent décrites. Par exemple, le 14 août 1918, alors qu'elle est en vacances à Rivière-duLoup avec ses filles Marie, Berthe et Jeanne, elle a une «belle et grande surprise». "Ce soir vers dix heures [...] l'on fit entrer au salon, mon cher mari avec notre Justine. Ils avaient envoyé une dépêche que nous n’avions pas reçue. Il y eut donc des cris de joie et d'étonnement, mon cœur battait bien fort ${ }^{48}$.»

Les battements de cour reviendront ailleurs comme descripteurs d'une joie intense. C'est le cas en 1904, alors qu'elle retrouve à Québec son mari revenant d'Angleterre. «Le cœur me battait bien fort [...] Ce fut une joie générale ${ }^{49}$.» C’est aussi le cas en juin 1908, lorsqu'elle retrouve son amie d'enfance Marie de Kersabiec ${ }^{50}$, à Saint-Étienne-de-Montluc, en Loire inférieure, au cours d'un voyage en France. Elle raconte: «Je me hâte de monter vers ma bien chère amie d'enfance la Comtesse de Kersabice qui se jette dans mes bras. Nos cœurs battaient de la même manière après 15 ans, quelle émotion de nous revoir.»

Bref, Marie-Louise Globensky valorise l'amour conjugal, maternel, familial et amical. Lorsqu'elle passe du temps avec les gens qu'elle aime, elle est en concordance avec ses valeurs et en accord avec la norme émotionnelle, selon laquelle le rôle principal des femmes est un rôle d'amour et de lien ${ }^{51}$. Ce faisant, elle se sent avancer dans le droit chemin, et elle ressent de la joie... Cela dit, dans sa représentation des joies d'amour et de liens, Globensky se pose en modèle sans faille de femme aux relations harmonieuses. Ce modèle, tout à fait lisse, n'est probablement pas complètement juste. En effet, il y a des indices de moments non harmonieux

47. Journal, 22 avril 1908.

48. Journal, 14 août 1918.

49. Journal, 26 août 1904.

50. Globensky écrit «Kersabice», mais il s'agit de Marie Sioc’han de Kersabiec, comtesse de Beaujeu, une amie d'enfance ayant épousé Yves de l'Escale en août 1893, à Saint-Étienne-de-Mont-Luc, en Loire inférieure. Marie de Kersabiec vivra la majeure partie de sa vie en France et y mourra en 1915.

51. Elle cite plusieurs sermons qui vont en ce sens. 
avec ses proches dans sa correspondance, où elle se préoccupe visiblement moins de l'image qu'elle laisse à la postérité ${ }^{52}$.

Par ailleurs, le fait que les joies d'amour et de liens soient valorisées pour les femmes bourgeoises n'est pas totalement innocent. En effet, en encourageant ces émotions, le discours normatif renforce l'adhésion de ces femmes à leur mission "féminine", ce qui rend bien service à la communauté. Les réseaux de liens affectifs entretenus par les femmes ne sont-ils pas la clé d'un système d'entraide et de soutien informel qui se déploie de façon extrêmement efficace dans les cas de maladie, de deuils et d'accidents, avant l'avènement de l'État-Providence?

\section{Joies spirituelles}

\section{Cérémonies religieuses}

La fête de Noël est souvent une occasion pour Globensky d'exprimer de la joie. Dans ses descriptions, son émotion culmine pendant la messe de Minuit, alors que la musique et la lumière concourent à la susciter. Alors, elle a l'impression d'être liée à plus grand qu'elle. La joie, il faut le dire, est l'émotion que la chrétienté associe à Noël ${ }^{53}$ et Globensky a très bien intériorisé cette norme. Le 24 décembre 1898, alors qu’elle a 49 ans, elle vit un Noël des plus heureux :

J'ai préparé aujourd'hui la crèche de Noël pour les enfants puis ce soir comme d'habitude il y eut du monde ici. À minuit, [...] [j]'allai avec Thaïs et Berthe au couvent de la Miséricorde, j'aime le recueillement dans une communauté. Nous eûmes une très belle messe, beau chant, très belle illumination puis surtout ce silence et cette ferveur qui édifie, là nous contemplions l'EnfantJésus avec des cœurs tout à lui il me semble, n'ayant rien pour nous distraire. Ce fut la première messe de minuit de ma petite Berthe, elle en fut ravie. La nuit était superbe, une température délicieuse. Au retour, nous nous mîmes à table au nombre de vingt-deux, une grande gaieté y fit durer la fête jusqu’à quatre heures ${ }^{54}$.

52. Dans une lettre à sa fille Blanche en novembre 1907, par exemple, Marie-Louise Globensky se plaint de son fils Louis, qui refuse de faire ses Pâques. «Il ne veut pas faire ses Pâques, il refuse encore de porter une médaille, quelle obstination désolante. Si tu savais combien je souffre de cela.» Lettre de Marie-Louise à Blanche, 10 novembre 1907, BAnQ Vieux-Montréal, Fonds Famille Landry, P155, S8, SS2, D6, 1975-00-027/52.

53. "L'évangile de $\operatorname{Luc}(2,10-13)$ relate qu'un ange annonça "une grande joie" aux bergers la nuit de Noël [...]», rappelle Jean Delumeau. Dès le Moyen Âge, montre-t-il, l'iconographie s'empare du thème qu'est la joie de Noël. Cette association entre la fête de Noël et l'émotion de la joie traverse les siècles. Jean Delumeau, Que reste-t-il du paradis? (Paris, Fayard, 2000), 226.

54. Journal, 24 décembre 1898. 
Les moments de grâce et de ferveur religieuse sont décrits avec emphase dans le journal, notamment parce qu'ils font partie des expériences précieuses de la vie pour Globensky, de celles dont elle veut témoigner. En les couchant dans son journal, elle les immortalise, ce qui est aussi une façon de confirmer, pour elle-même, qu'elles ont bel et bien eu lieu. De confirmer aussi, pour ceux qui liront le journal, la sincérité de sa foi. Et de leur transmettre le désir de vivre de telles émotions par la foi.

La joie étant une émotion "attendue» le soir de Noël, son absence, quelle qu'en soit la raison, est vécue douloureusement par Globensky. À Noël 1907, par exemple, la diariste est plongée dans une angoisse profonde parce que son fils Alexandre est malade et hospitalisé55. Sans joie, elle décide de ne pas aller à la messe de Minuit. L'entrée qu'elle écrit dans son journal dit que la joie des autres lui fait mal et se termine par une phrase qui appelle Jésus, celui par qui la joie arrive, à ramener cette émotion dans son foyer:

Seule je reste contre mon habitude n'ayant pas le courage de me rendre à cette fête si joyeuse de Noël. L'émotion me suffoquerait [...]. Quel changement cette année à cette époque toujours si gaie pour nous, où une nombreuse jeunesse entourait le souper de famille et qui chantait ensuite gaiement les cantiques de Noël. Mon pauvre cœur se brise à ce silence profond, mon pauvre Alex, le plus gai de la bande est absent, malade. L'épreuve est voulue par Dieu, je me soumets mais je souffre cruellement, la gaieté me fait mal. Oh! Aimable Jésus, vous qui apportez la joie, faites-la renaitre sous notre toit ${ }^{56}$.

Il arrive que des êtres chers soient le prétexte d'un office religieux; les émotions sont alors d'autant plus fortes. C'est le cas lors de la première communion de la petite-fille de Globensky, Marie Gérin-Lajoie ${ }^{57}$, le 11 mai 1900, dans la chapelle de l'académie Saint-Urbain ${ }^{58}$. Globensky décrit la cérémonie et ses émotions :

La petite chapelle ornée de ses plus belles parures est remplie de parents recueillis en attendant l'arrivée des jeunes communiantes. Tout-à-coup [sic] l'on voit apparaitre comme une vision céleste, le cortège de ces petits anges vêtus de blanc et portant à la main un cierge allumé. Ma chère petite Marie est la première dans le rang, nous pouvons alors la suivre de près. La musique

55. Nous ignorons toujours de quelle maladie il est atteint, car Globensky ne la nomme jamais. Mais en raison du tabou qu'il semble y avoir autour d'elle, nous penchons pour une maladie mentale ou une cure contre l'alcoolisme.

56. Journal, 24 décembre 1907.

57. Marie Gérin-Lajoie (1890-1971), future fondatrice de l'Institut Notre-Dame-du-Bon-Conseil.

58. Située au 456 Saint-Urbain (entre Milton et Prince Arthur). 
fait entendre ses accords harmonieux et bientôt le prêtre commence le saint sacrifice. [...] Comment redire l'émotion de nos âmes à ce moment; jamais la parole humaine ne le redira, les âmes seulement qui ont reçu ce don de Dieu, de ses faveurs intimes en comprendront toute la valeur. C'est ma petitefille qui va recevoir pour la première fois ce Jésus de l'Eucharistie, notre Dieu, notre Père, notre Tout ${ }^{59}$.

Marie-Louise Globensky, ici, est fière et reconnaissante de voir sa petitefille aimée s'engager sur le chemin de la foi chrétienne et catholique, pour elle voie sacrée vers le bonheur éternel. Elle a aussi peut-être le sentiment d'avoir bien accompli son devoir de grand-mère, ayant, par sa piété, montré le chemin.

Dans toutes les entrées présentant des moments de grâce qui se déroulent à l'église, Globensky s'attarde à décrire la beauté, la lumière, la musique, tout ce qui est «brillant», «harmonieux», «féérique» ou "resplendissant». Ces éléments, préfigurant le paradis ${ }^{60}$, ne sont pas là par hasard. Ils ont pour fonction précise de susciter et d'intensifier ces émotions d'élévation que Globensky ressent. Au carrefour de l'intime et du social, les émotions révèlent toujours autant sur les individus que sur les sociétés ${ }^{61}$. Ici, on le voit, la joie encouragée par des moyens précis, rend plus belle, plus agréable, plus «habitable» la vie d'une personne, mais elle permet aussi à une institution dominante dans la société, l’Église catholique, de consolider son influence en fidélisant ses ouailles.

\section{Dans la nature}

Parfois, l'écrin dans lequel se déploie la joie est plus vaste que celui d'une église. Globensky expérimente des états de grâce et d'émerveillement alors qu'elle se trouve dans la nature, devant un coucher de soleil, un ciel étoilé ou un paysage enchanteur, souvent en vacances à Kamouraska, à Rivière-du-Loup, à Saint-Irénée ou dans les Hautes-Laurentides, où la famille Lacoste possède des terres. Le 28 juillet 1897, alors qu'elle vient de vivre un de ces moments sur la route de Kamouraska, elle écrit:

59. Journal, 11 mai 1900.

60. La musique, la lumière, les fleurs, les odeurs sont considérées comme des attributs du ciel. J. Delumeau, op. cit., 226.

61. «Entre l'individuel et le collectif, entre l'intime et l'exhibé, les émotions sont ressenties individuellement mais données à voir ou partagées collectivement. Les individus se trouvent pris entre la singularité de leur autonomie et la norme collective, entre des communautés qui règlent comportements et langages et les capacités singulières d'intériorisation. L'espace privé n'est ainsi jamais l'univers d'une expression individuelle absolument libre ", Anne-Claude Ambroise-Rendu et al., Émotions contemporaines, XIX ${ }^{e}$-XXI ${ }^{e}$ siècles (Paris, Armand Colin, 2014), 15. 
Nous ne sommes arrivés ici qu'à une heure. Mon mari et monsieur Angers nous attendaient à la porte car la soirée était charmante. Depuis longtemps je n'avais vu un ciel si étoilé. Je ne me lassais pas d'admirer un pareil spectacle et de bénir Celui qui nous réjouit de telles merveilles. Comme le ciel doit être beau, puisque nous nous sentons dans le ravissement en le regardant de si $\operatorname{loin}^{62}$.

À certains moments, le ravissement devant la nature mène à une sorte d'extase spirituelle, comme le 24 juillet 1918, à Rivière-du-Loup, où l'on voit réapparaître les battements de cœur des joies intenses des retrouvailles, cités plus haut. Cette fois, ce sont des battements de cœur de gratitude envers celui qui crée une si belle nature.

Nous avions ce soir, un coucher de soleil ravissant, ses derniers feux ranimaient toute la nature de mille teintes éblouissantes qui nous charmaient. Un peu plus tard nous fûmes attirées par la lune sortant derrière les montagnes dans sa plénitude superbe. La voilà radieuse. Que c'est une jouissance de pouvoir contempler ainsi la belle nature sans aucun obstacle devant soi. C'est alors que l'on sent en son âme un amour invincible pour le Créateur de si grandes choses. C'est une parcelle des beautés du ciel qu'il nous laisse entrevoir afin de le désirer. Oui, mon Dieu, sentez battre mon cœur qui s'exalte de reconnaissance et faites que je vive pour le mériter et que j’y amène tous ceux que vous m'avez confiés ${ }^{63}$.

La nature, on le voit, agit comme un déclencheur de la grâce. Sa beauté évoque le paradis et les émotions ressenties devant elle, deviennent une confirmation de la foi.

La foi a été, dans l'expérience humaine de Globensky, une importante source de joie. Elle a donné sens à sa vie, lui a offert des moments de réconfort, d'émerveillement, de gratitude et d'espérance. Globensky a voulu témoigner de ses joies spirituelles, pour immortaliser ces émotions, mais aussi pour convaincre ses descendants de la suivre sur le chemin du paradis... "Que la foi est belle, que l'espérance est admirable, sublimes vertus, soutenez-nous toujours ${ }^{64}{ }^{\prime}$, écrivait-elle. On voit également que l’Église catholique entretenait volontiers ces émotions de joie ressenties autour de la foi. Parce que ces émotions, plus que tout peut-être, attachaient les fidèles à leur Église. 


\section{Joies de s'engager dans la société}

\section{Tenir son rôle de bourgeoise}

Marie-Louise Globensky a une vie sociale remplie. Elle prend à cœur les obligations sociales qui viennent avec son rang: recevoir, accompagner son mari dans des réceptions, être reçue chez des amies pour le lunch ou le thé, superviser la jeunesse lors des bals, etc. Si ces activités sociales lui donnent beaucoup de plaisir et qu'elle ne s'en cache pas, elles lui apportent aussi une joie plus profonde: un sentiment de s'accomplir à travers le rôle social qui lui a été imparti.

Les mercredis, pendant des décennies, Marie-Louise Globensky «tient son jour ", c'est-à-dire qu'elle reçoit, dans sa maison de la rue Saint-Hubert, des personnalités de la bourgeoisie montréalaise. Globensky décrit ces réceptions dans des termes joyeux, par exemple, le mercredi 27 janvier 1897 : «[...] nous avons eu beaucoup de monde, la gaité et l'entrain en ont fait une charmante soirée $\left.{ }^{65}\right)$.

Si Marie-Louise Globensky éprouve de la fierté et une certaine satisfaction à jouer son rôle d'hôtesse, elle éprouve des sentiments similaires lorsqu'elle est reçue, notamment lorsqu'elle accompagne son mari dans des réceptions. Elle semble fière de dire qu'elle a côtoyé des gens qu'elle juge importants. Le 15 janvier 1907, par exemple, elle écrit:

Ce soir, je vais dîner avec mon mari chez lord Strathcona, je suis accompagnée à table par Son Excellence le Gouverneur général. Il est très aimable, mon voisin de droite est Sir Thomas Shaughnessy. La table est toute étincelante de lumières et parfumée de fleurs splendides. Une soixantaine de convives, toilettes superbes, conversation très animée, nous nous amusons fort bien. De là, nous nous rendons tous au bal de charité au Windsor dont je suis une dame patronnesse. J'ai l'honneur de causer beaucoup avec lady Grey, qui est toujours fort charmante. Je vais souper avec Sir Montagu Allan. Nous avons passé une fort agréable soirée ${ }^{66}$.

Il est intéressant de remarquer la présence, comme dans les joies spirituelles, des fleurs et de la lumière. Ces éléments sont utilisés pour créer

65. Journal, 27 janvier 1897. La Revue moderne fait mention de ces réceptions dans un article paru à l'occasion du décès de Globensky en 1919: «Hospitalière comme elle savait l'être, à la façon du temps passé, elle reçut dans son salon, pendant au moins 30 ans, tout ce que le Canada français a connu de célébrités... en herbe ou de personnes au sommet de la gloire», cité dans Pierre Vennat, «Il y a 150 ans naissait Lady Lacoste, doyenne d'une famille de "femmes d'œuvres" ", La Presse, dimanche 27 juin 1999, p. A6.

66. Journal, 15 janvier 1907. 
de la beauté et ainsi renforcer les émotions de bien-être, de confort, de fierté des élites qui se rassemblent ${ }^{67}$.

C'est aussi vrai lors d'un dîner chez sir Montagu Allan en l'honneur de la visite du prince japonais Fushimi:

C'est une fête princière, 64 convives, table recouverte de fleurs et de bougies, mets exquis, délicieuse musique. Je fus conduite à table par l'interprète du prince qui parle l'anglais et qui est bien aimable. Après le dîner, chacun d'aller voir la splendide illumination dans les jardins, des chaînes de lumières électriques traversant dans toutes les directions ces parcs magnifiques, donnaient un aspect féérique. [...] La soirée fut très animée et nous avons rapporté un charmant souvenir ${ }^{68}$.

Globensky mentionne ici les plaisirs de la nourriture, ce qui est rare dans son journal ${ }^{69}$. La gourmandise est un péché capital selon la religion catholique, mais elle semble acceptable, voire bien vue, dans un contexte mondain à l'intérieur de la grande bourgeoisie bilingue montréalaise.

Lors de certains événements, comme des bals ou des «skating party», Marie-Louise Globensky joue un rôle de supervision. Sa présence, comme celle d'autres dames bourgeoises respectées, donne un sceau de pureté morale à ces rencontres où les plaisirs sensoriels, notamment ceux de la danse, pourraient comporter des risques. Le 8 janvier 1907, elle raconte:

[...] le soir j'allais patronner le bal des jeunes gens au Windsor. J'avais pour compagnes Mmes L.R. Masson, R. Dandurand et L.J. Forget. La musique entraînante enlevait l'enthousiasme de la jeunesse fraîche et riante. Tout se passa très bien. Le souper délicieux, charmante société ${ }^{70}$.

En janvier 1900, elle décrit un «skating party» qu’elle organise pour les jeunes:

[...] la soirée a été charmante. À peu près quatre-vingts personnes se sont rendues à l'appel. Le rink était illuminé aux lanternes chinoises ce qui produisait un effet féérique. Les couples se balançant gracieusement en faisant le tour semblaient jouir d'un plaisir particulier. Aussi restèrent-ils bien long-

67. Le lien entre lumière et émotions est analysé par Nicolas Kenny dans un récent article sur l'éclairage électrique de Montréal et de Bruxelles à la fin du XIX siècle. N. Kenny, «City Glow : Streetlights, Emotions, and Nocturnal Life, 1880s-1910s ", Journal of Urban History, 1, 24 (2015): 1-23.

68. Journal, 9 juin 1907.

69. Le journal est par ailleurs complètement silencieux sur la sexualité, ce qui indique combien les plaisirs des sens étaient tabous.

70. Journal, 8 janvier 1907. 
temps dehors, ils entrèrent alors pour faire quelques tours de valse, puis l'on babilla très longtemps en prenant quelques rafraichissements $[\text { sic }]^{71}$.

Dans ces soirées mondaines où elle côtoie l'élite de sa société, à la fois anglophone et francophone, protestante et catholique, Globensky se sent utile et à sa place. Elle accomplit son devoir d'épouse en accompagnant son mari et elle contribue à la vitalité de la communauté bourgeoise. Lors des bals pour la jeunesse et des "skating party», elle vit par procuration l'excitation joyeuse de la jeunesse. Elle vit peut-être aussi une certaine nostalgie de ne plus faire partie de cette jeunesse. Mais on sent surtout le plaisir qu'elle a à jouer auprès de ces jeunes un rôle de supervision, de chaperon, de modèle. En l'assumant, elle contribue par ailleurs à la reproduction sociale de la classe bourgeoise (car ces événements sont des lieux de sociabilité et de rencontre pour les jeunes couples), donc à la survie de modes de vie auxquels elle est attachée.

Dans ces plaisirs mondains, l'appartenance de classe semble prévaloir sur la religion, le genre, l'appartenance ethnique et les idéaux politiques. En effet, les gens de la grande bourgeoisie montréalaise, qu'ils soient anglophones ou francophones, femmes ou hommes, catholiques ou protestants, conservateurs ou libéraux semblent former une communauté émotionnelle particulière ${ }^{72}$, avec ses propres codes, qui entrent parfois en contradiction avec la morale austère catholique (notamment dans l'appréciation des mets exquis), ce qui ne semble pas trop perturber Globensky. Les émotions de joie, de réconfort, de fierté, qui sont entretenues lors de ces rendez-vous entre gens de la grande bourgeoisie, jouent un rôle de consolidateur de leur identité de classe et contribuent à la perpétuation des rapports de classe dans la société.

La vie sociale de Globensky se passe aussi parfois sans son mari. En effet, régulièrement, elle est invitée à luncher ou à prendre le thé, chez des dames de la haute société, francophones comme anglophones, d’allégeance conservatrice (comme elle) ou libérale ${ }^{73}$. Par exemple, en janvier 1899, plusieurs

71. Journal, 22 janvier 1900.

72. Selon Barbara Rosenwein, les individus appartiennent à plusieurs communautés émotionnelles, qui peuvent se superposer, se juxtaposer, s'opposer et qui évoluent. Marie-Louise Globensky, par exemple, fait partie de la grande communauté émotionnelle de la bourgeoisie francophone et catholique montréalaise, mais aussi de la grande bourgeoisie bilingue de la cité, où les normes, en ce qui concerne les émotions, ont leurs spécificités.

73. Dans sa thèse de doctorat, Élizabeth Kirkland montre qu'avant la Première Guerre mondiale, les femmes de la grande bourgeoisie anglophone et francophone ont su faire fi des divisions ethniques et religieuses et se rallier sur la base de leur appartenance de classe et de genre, afin de se donner plus d'influence et de pouvoir dans la société. E. Kirkland, Mothering Citizens. Elite Women in Montreal (1890-1914), thèse de doctorat (histoire), Université McGill, 2011. 
événements sont organisés autour de la visite à Montréal de lady Laurier (Zoé Lafontaine, femme du premier ministre libéral Wilfrid Laurier). "Aujourd'hui je suis allée à un lunch charmant chez madame Stephens, en l'honneur encore de Lady Laurier, nous étions quatorze dames ${ }^{74}$ ", écrit Globensky. Deux jours plus tard, elle écrit encore: «Je suis allée à un très beau lunch chez Lady Van Horne pour rencontrer Lady Laurier, vingt-deux convives, tout était exquis. [...] Notre visiteuse, femme du premier ministre, nous tient constamment en fêtes très agréables ${ }^{75}$.»

Le plaisir ressenti par Marie-Louise à prendre sa place comme femme bourgeoise dans la société montréalaise est indissociable de son acceptation de l'ordre social établi. Même si, à quelques reprises dans son journal, Globensky réfléchit sur la division de la société entre pauvres et riches, même si elle est consciente de son statut privilégié, elle cautionne complètement la division de la société en classes sociales, allant jusqu’à reprendre à son compte l'idée véhiculée par l'Église disant que cette ségrégation est l'œuvre de $\mathrm{Dieu}^{76}$, pour permettre aux privilégiés d'exercer la charité :

Cet après-midi, nous avons eu une très jolie conférence à la Providence [...]. Il nous parla des idées socialistes qui existent aujourd'hui et nous montra qu'il est nécessaire qu'il y ait des pauvres et des riches afin de s'entr'aider et de pratiquer la charité chrétienne. Dieu a voulu qu'il en fut ainsi puisqu'il a dit: Vous aurez toujours des pauvres parmi vous ${ }^{77}$.

Ce discours, elle n'a évidemment pas intérêt à le remettre en cause et, pas plus qu'une autre diariste bourgeoise de son temps, Joséphine Marchand-Dandurand (libérale et plus progressiste que Globensky sous plusieurs aspects, née 12 ans après elle), elle ne le questionnera ${ }^{78}$.

74. Journal, 23 janvier 1899.

75. Journal, 25 janvier 1899.

76. On retrouve aussi ce discours chez les protestants de New York au début du XIX ${ }^{\mathrm{e}}$ siècle. Voir Christine Stansell, City of Women. Sex and Class in New York 1789-1860 (Chicago, University of Illinois Press, 1987), 31-32.

77. Journal, 25 février 1907.

78. Joséphine Marchand-Dandurand accepte aussi très bien la division de la société en classes, se montrant souvent méprisante envers les classes populaires. Par exemple, elle accuse les pauvres d'être imprévoyants en matière d'économie, de se payer du luxe au mépris de l'épargne, elle déplore que les filles des familles pauvres apprennent la musique alors que ce n'est d'aucune utilité pratique (elles devraient plutôt apprendre à broder et à tricoter), et elle a un discours méprisant envers les domestiques, "fléau du siècle», qui font "tout de travers». Voir: Sophie Doucet, Joséphine Marchand-Dandurand ou "Le Laurier féminin ". Une journaliste féministe, moderne, libérale et nationaliste (1861-1925), mémoire de maîtrise (histoire), Université de Montréal, décembre 2003, 71. 


\section{Aider les pauvres}

Si Globensky ne semble pas se sentir coupable de faire partie des privilégiés alors que tant de gens vivent dans la misère ${ }^{79}$, c'est parce qu'elle remplit bien ses devoirs de charité. Et qu'elle le fait de la «bonne façon» c'est-à-dire comme l'Église le prescrit. «Il ne suffit pas de donner de son or sans se déranger, il faut au contraire se donner soi-même en s'associant aux bonnes œuvres ${ }^{80}$ », écrit-elle. Ailleurs, elle est plus précise : «Dans l’après-midi l’instruction fut sur nos devoirs de dames de Charité, puis la manière de la faire. Non seulement donner du pain et des vêtements, mais visiter les pauvres et leur apporter de l'encouragement et de l'espérance ${ }^{81}$.»

Globensky a fortement intériorisé cette vision catholique du rôle des femmes dans la charité. C'est ce qu'elle met en ouvre au quotidien, notamment avec ses sœurs Coralie (Masson, puis Taschereau) et Élodie (Garneau), en visitant les "déshérités», en cousant pour eux, en parrainant certains d'entre eux, en participant à la recherche d'un toit pour des malades et des orphelins. C'est celle qu'elle a incarnée jusqu'au bout de sa vie. En effet, la veille même de sa mort, dans l'ultime entrée de son journal intime, Marie-Louise Globensky se réjouit d'avoir pu trouver un foyer à quatre petits orphelins ${ }^{82}$.

Ces bourgeoises ont, à l'égard des pauvres, une attitude condescendante et parfois moraliste ${ }^{83}$. Cette attitude se ressent dans plusieurs entrées du journal de Globensky.

$[\ldots]$ à trois heures je me suis rendue au Couvent de la Providence pour assister à cette si touchante cérémonie de la distribution des vêtements aux pauvres. Comme il fait bon de réjouir ces déshérités de la fortune. Un grand nombre de femmes et d'enfants se groupaient près des tables, les dévorant de leurs regards. De gros paquets de lingerie se donnaient par numéro, puis ensuite l'on distribua des gâteaux, bonbons et jouets aux enfants qui ne se possédaient pas de joie ${ }^{84}$.

79. Voir notamment: Bettina Bradbury, Familles ouvrières à Montréal. Âge, genre et survie quotidienne pendant la phase d'industrialisation (Montréal, Boréal, 1995); Terry Copp, The Anatomy of Poverty, The Condition of the Working Class in Montreal 1897-1928 (Toronto, McClelland and Stewart, 1974).

80. Journal, 23 mars 1917.

81. Journal, 28 septembre 1910.

82. "Quel bonheur j’éprouve d'annoncer cette bonne nouvelle... ", Journal, 10 décembre 1919.

83. Magda Fahrni fait référence à la condescendance et au moralisme des dames patronnesses visitant les pauvres lors de l'épidémie de grippe espagnole de 1918, en mentionnant que ces attitudes n'excluaient pas la compassion. "Elles sont partout...": les femmes et la ville en temps d'épidémie, Montréal, 19181920 ", Revue d'histoire de l'Amérique française, 58, 1 (été 2004): 81.

84. Journal, 19 novembre 1904. 
Ainsi, elle a l'impression de distribuer de la joie. C'est encore plus clair dans cette entrée où elle raconte une visite à son "petit protégé», un enfant nommé René Hébert, à l’hôpital des Incurables:

L'enfant est mieux, le médecin a de l'espoir. Il était heureux comme un prince avec ses bonbons et ses bibelots que nous lui avions apportés. Qu'il faut peu parfois pour donner du bonheur. Et quelle joie en notre âme lorsqu'il nous est donné de rendre le sourire à celui qui souffre ${ }^{85}$.

Cette impression de distribuer de la joie lui procure en retour fierté et satisfaction. Le mardi 27 décembre 1898, elle évoque ces sentiments:

Nous avons eu aujourd'hui notre dîner annuel de l'Hôpital Notre-Dame [...]. Quel touchant spectacle toujours de voir ces pauvres malades, à qui nous apportons quelques consolations ce jour-là. Comme nous nous sentons heureuses et comme nous sommes bien payées de nous déranger pour eux. Que de misères humaines sont réfugiées dans ces asiles.

Par ailleurs, elle ne cache pas que tout cela n'est pas complètement gratuit, car Dieu saura récompenser ceux qui sont charitables:

$[\ldots]$ nous avons eu un très beau sermon sur la charité. Qu'il est consolant de savoir que Dieu récompense si bien l'aumône faite au moindre de ses membres souffrants. Rachetons toutes nos iniquités, achetons le ciel puisque nous en connaissons le moyen; qui donne aux pauvres prête à Dieu, il nous le rendra au centuple ${ }^{86}$.

Bref, qu'elle tienne son rang à travers des réceptions mondaines ou qu'elle fasse la charité aux pauvres, Globensky éprouve des émotions de la famille de la joie (notamment de la satisfaction, de la fierté) à s'accomplir dans la société à l'intérieur des limites qui sont celles imposées aux femmes. Elle ne semble pas souffrir de ces limites. En effet, en jouant la partition qui lui a été attribuée, en agissant comme «doit» le faire une femme bourgeoise franco-catholique, elle est en harmonie avec ses valeurs et avec la norme émotionnelle, elle a l'impression d'accomplir sa mission et d'être utile à sa société, ce qui la satisfait.

Par ailleurs, il est intéressant et révélateur de mettre en comparaison les joies des pauvres et la joie de la diariste mises en représentation dans ce journal. Les joies des pauvres sont naïves, enfantines, presque primitives (ils «dévorent du regard» les paquets de vêtements, ils ne «se possèdent plus de joie»), alors que la joie de la diariste est profonde et géné- 
reuse, réfléchie et digne, c'est une joie de devoir et de foi. En présentant cette émotion de façon si contrastée, Globensky montre que la joie, cette émotion agréable qui rend la vie sur terre plus belle et plus «habitable», peut être par ailleurs un puissant marqueur d'identité de classe. Consciemment ou non, Globensky l'utilise pour creuser la distance entre elle et les "pauvres». Ainsi, cette émotion est au service de l'appartenance de classe et contribue au statu quo dans les rapports sociaux.

\section{CONCLUSION}

Nous avons observé que la joie est une émotion extrêmement présente dans la représentation que fait Marie-Louise Globensky de son paysage émotionnel. La joie, visiblement, était bien perçue dans la bourgeoisie franco-catholique montréalaise du tournant du $\mathrm{XX}^{\mathrm{e}}$ siècle, dans la mesure où elle était causée par des événements ou des situations moralement acceptables. Dans ce cas, elle était tout aussi valorisée, en fait, que la tristesse et l'angoisse, qui rappelaient le passage de Jésus sur terre. Si toutes ces émotions «vertueuses» faisaient de l'humain ce qu'il était et ouvraient les portes du paradis, la joie avait la particularité de donner un avant-goût du «bonheur éternel» et il était bien vu de la cultiver.

Lorsqu'elle aime, lorsqu'elle prie ou se «connecte» à Dieu, lorsqu'elle s'engage socialement de la «bonne» façon, Marie-Louise Globensky respecte ses valeurs et la norme émotionnelle et a l'impression de marcher dans la bonne direction, ce qui lui fait ressentir et exprimer de la joie. En effet, la joie exprimée de Globensky s'accorde avec son but ultime, qui est d'aller au ciel à la fin de ses jours (et d'y retrouver ceux qu'elle aime). Elle montre aussi le chemin du paradis à ses descendants. Cette analyse laisse voir l'impact particulièrement grand de la religion ou du système de croyance sur le vécu émotionnel d'un individu.

Par ailleurs, si la joie a sans aucun doute rendu l'expérience humaine de Marie-Louise Globensky plus agréable, nous avons montré que le rôle de cette émotion dans la société est plus complexe. Les joies "valorisées » par les discours prescriptifs et que ressent Globensky sont effectivement au service de certaines forces en présence dans cette société. Les joies d'amour et de lien renforcent l'adhésion des femmes à leur rôle affectif et servent le statu quo dans les rapports de genre. Les joies spirituelles tiennent les fidèles attachés à leur religion et favorisent l'hégémonie de l'Église catholique. Les joies d'engagement social augmentent la distance qui sépare les riches et les pauvres et sont au service du statu quo dans les rapports de classe. Toutes ces joies - qui ne sont pas moins sincèrement 
éprouvées - semblent sciemment entretenues et leur instrumentalisation éclaire les rapports de force à l'œuvre dans la société montréalaise du tournant du $\mathrm{XX}^{\mathrm{e}}$ siècle.

Marie-Louise Globensky est une femme de son temps qui a fortement intériorisé les discours prescriptifs en ce qui concerne les émotions (qui acceptent la joie et la souffrance, tant qu'elles sont vertueuses) et qui ne remet pas l'ordre social en question. En effet, comme elle trouve à s'accomplir et ressent suffisamment de joie à l'intérieur de la sphère qui lui est assignée comme femme bourgeoise, elle n'est pas portée à demander des changements. D’ailleurs, il est intéressant de noter que le journal de Globensky ne comprend à peu près aucune trace de colère. Ce silence n'indique pas qu'elle n'en a pas ressenti, mais que cette émotion était si mal vue chez une femme bourgeoise, devant accepter le sort que Dieu lui avait réservé, qu'il était impensable de la représenter dans son journal. Il en sera autrement, une génération plus tard, pour sa fille Marie GérinLajoie, dont la sobre colère, exprimée dans ses écrits privés et publics ${ }^{87}$, servira le combat pour les droits des femmes.

87. Voir le journal intime de Marie Gérin-Lajoie (BAnQ Vieux-Montréal, P783, S2, SS1), 2007-10$005 / 1$, P2 / A04 et ses articles dans La Bonne parole. 